\title{
Self-rated disability in first treated episode of psychosis: A 1-year follow-up study
}

\author{
Carmen Simonsen ${ }^{\mathrm{a}, \mathrm{b}}, *$, Ann Faerden ${ }^{\mathrm{a}}$, Torill Ueland ${ }^{\mathrm{a}}$, Anja Vaskinn ${ }^{\mathrm{a}}$, Thomas Bjella ${ }^{\mathrm{a}}$, Ole Andreassen ${ }^{\mathrm{a}}$, \\ Kristin Lie Romm ${ }^{\mathrm{a}, \mathrm{b}}$, Ingrid Melle ${ }^{\mathrm{a}}$ \\ ${ }^{\text {a }}$ NORMENT, KG Jebsen Centre for Psychosis Research, Division of Mental Health and Addiction, Oslo University Hospital and Institute of Clinical Medicine, University of Oslo, Norway \\ ${ }^{\mathrm{b}}$ Early Intervention in Psychosis Advisory Unit for South East Norway, Division of Mental Health and Addiction, Oslo University Hospital, Norway
}

\section{A R T I C L E I N F O}

\section{Introduction}

Clinical symptoms and functional impairment are core features of psychotic disorders, with functional impairment being present already in first episode psychosis [1-3]. Functioning is often measured objectively with clinician-rated scales of global functioning measured in a single construct, or self-rating scales of social functioning measuring the frequency of various social activities. These measures are useful in outcome studies focusing on illness signs, with the absence or low levels of symptoms and functional impairment often referred to as clinical recovery [4]. However, there is an increasing effort to capture the

\footnotetext{
* Corresponding author at: Early Intervention In Psychosis Advisory Unit for South East Norway, Oslo University Hospital, Gaustad sykehus, Sognsvannsveien 21, Oslo, Norway.

E-mail addresses: c.e.simonsen@medisin.uio.no (C. Simonsen),

ann.faeren@medisin.uio.no (A. Faerden), torill.ueland@medisin.ui.no (T. Ueland), anja.vaskinn@medisin.uio.no (A. Vaskinn), thomas.bjella@medisin.uio.no (T. Bjella), o.a.andreassen@medisin.uio.no (O. Andreassen), k.l.romm@medisin.uio.no (K.L. Romm), Ingrid.melle@medisin.uio.no (I. Melle).
}

personal experience of illness and recovery, including the subjective experience of improved life satisfaction and participation in life, despite illness signs, often referred to as personal recovery [5]. Subjective life satisfaction in general and subjective experience of difficulties related to more specific areas of everyday activities and participation in life has been studied in psychotic disorders [6,7], although far less frequently than symptomatology and global functioning.

The WHO developed a conceptual framework for measuring health and disability across different cultures and settings, the International Classification of Functioning, Disability and Health (ICF; [8]). In the ICF framework, functioning and disability are recognised as outcomes of interactions between health conditions and contextual factors (environmental or personal). This implies that environmental facilitation can reduce the degree of disability from a medical disorder. In psychotic disorders this could involve adequate treatment, social services, job opportunities or the combat of stigmatising attitudes. The framework divides functioning into 6 life domains: understanding and communicating, getting around, self-care, life activities, getting along with people, and 
participation in society, with the two latter domains being social in nature. A self-rating measure called the World Health Organization Disability Assessment Schedule 2.0 (WHODAS 2.0; [9]) was developed to assess the activity limitations and participation restrictions experienced on these 6 major life domains.

Few studies of psychotic disorders have used the WHODAS 2.0. Yet the measure has been found reliable and valid for use with psychotic disorders in cross-sectional studies; with out patients, older patients and long-term patients showing greater social disability than healthy controls [10-12]. These studies found fewer activity limitations and participation restrictions self-reported by WHODAS 2.0, than rated by clinicians. Moreover, although WHODAS 2.0 was related it was distinct and complementary, to a self-rated measure of life satisfaction. In sum, they found that self-rated disability was more closely related to depressive symptoms and life satisfaction, and less so to psychotic symptoms, cognitive functioning and global functioning.

To our knowledge there is only one study applying WHODAS 2.0 to individuals with first episode psychosis, thus reducing the potential influence of illness duration and treatment [13]. Chudleigh et al. [13] found that compared to a healthy control group, the first episode psychosis sample had both lower quantitative ratings of social functioning (measured by the Social Functioning Scale) and higher qualitative self-rated experience of disability across the 6 domains (measured by WHODAS 2.0). Moreover, they reported that disability was equal in an at-risk group of individuals, thus preceding psychosis onset, which is in line with another at-risk study reporting that the social domains predict development of psychosis [14]. Finally, Chudleigh et al. [13] found that self-rated disability was primarily related to depression and social anxiety and less so to positive psychotic symptoms.

Global functioning measured with GAF-F has been reported to improve in first episode psychosis across the first year after treatment start and to be related to psychotic symptomatology $[2,15]$. Satisfaction with life in general has also been reported to improve across the first 2 and 10 years, yet to be most closely related to depression in first episode psychosis and long-term schizophrenia [6,16,17]. As mentioned above self-rated disability has also been found to be most closely related to depression [13], but it remains to be studied longitudinally in first episode psychosis.

Based on the above research with relatively small sample sizes, level of clinically significant self-rated disability in first episode psychosis as well as its longitudinal development remains unclear. Moreover, how self-rated disability relates to clinician-rated global functioning, selfrated life satisfaction and symptomatology in first episode psychosis requires investigation. Finally, the best predictors of self-rated disability in first episode psychosis remain to be identified. An improved understanding of self-rated disability in first episode psychosis could guide the development of early treatment and environmental facilitation compensating for symptoms and loss of functioning. The present paper therefore has the following aims:

1. Investigate self-rated disability in first treated episode of psychosis across the first year of treatment.

2. Investigate the relationship between self-rated disability and clinician-rated global functioning, self-rated life satisfaction and symptomatology in first treated episode of psychosis at 1-year follow-up.

3. Investigate premorbid and baseline predictors of self-rated disability in first treated episode of psychosis at 1-year follow-up.

\section{Methods}

\subsection{Participants}

The participants were part of a planned follow-up study, recruited between 2003 and 2014 from in-patient and out-patient psychiatric units in all major hospitals in Oslo, Norway, covering a catchment area of about 485,000 inhabitants. Participant recruitment was carried out through a close collaboration between the treating clinicians in the various psychiatric units (identifying suspected psychotic disorders) and the TOP assessment team (carrying out the structured diagnostic assessment). Participants were recruited into the study within 52 weeks following the onset of adequate treatment for a psychotic disorder (i.e. antipsychotic medication in adequate dosage for $>12$ weeks or until remission, or hospitalisation in psychiatric wards treating psychosis). Thus the sample is more precisely termed first treated episode of psychosis rather than first episode psychosis. However, we argue that our sample is comparable to a number of other first episode psychosis samples, which for example include participants with a recent first diagnosis in the psychosis spectrum [18]. Inclusion criteria for the present study was a 1-year follow-up diagnosis (Diagnostic and Statistical Manual of Mental disorders-IV (DSM-IV) (American Psychiatric Association, 1994) belonging to the broad schizophrenia spectrum, defined as schizophrenia, schizophreniform disorder, schizoaffective disorder (constituting "schizophrenia spectrum disorder") or delusional disorder, brief psychotic disorder and psychosis NOS (constituting "other psychosis"). Major depressive disorder (MDD) with psychotic symptoms meeting the A-criterion for schizophrenia was also included at baseline as this condition can develop into diagnoses from the broad schizophrenia spectrum. Our attrition rate calculations from baseline to 1-year follow-up therefore include participants with MDD at baseline. A total of 298 participants with broad schizophrenia spectrum and MDD with psychosis were included at baseline, with 185 of them (62\%) being reassessed at 1-year followup. When excluding the participants fulfilling criteria for MDD at one year follow-up (17 participants) the total sample consisted of 168 participants within the broad schizophrenia spectrum. A number of these participants were excluded due to missing WHODAS 2.0 data (9 participants missing at both time points, 13 participants missing at baseline and 27 missing at 1-year follow-up). One participant was excluded because of missing PANSS scores and 4 participants were excluded due to IQ below 70. The final sample therefore consisted of 115 participants (See Fig. 1). There were no significant baseline demographic or clinical differences between the participants that completed reassessment at 1-year follow-up and those that did not. Likewise there were no significant demographic or clinical differences between the broad schizophrenia spectrum participants reassessed at 1-year follow-up with missing WHODAS 2.0 data and those in the final sample.

Exclusion criteria for first treated episode of psychosis participants were hospitalised head injury, neurological disorder, unstable or uncontrolled medical condition that interferes with brain function, IQ below

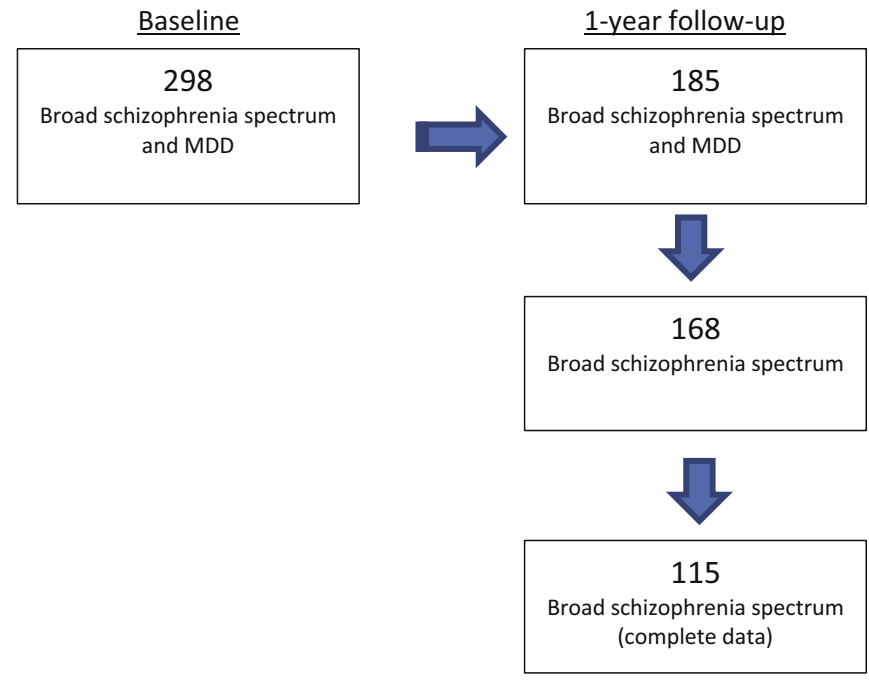

Fig. 1. Flow-chart - Attrition rate. 
70, and age outside the range of 18-65 years. The study was approved by the Regional Committee for Medical Research Ethics and the Norwegian Data Inspectorate. Data was obtained in compliance with the regulations of our institutions. After complete description of the study all participants gave written informed consent.

\subsection{Clinical assessment}

Structured clinical assessment was carried out by trained investigators consisting of clinical psychologists and medical doctors. Diagnosis was based on Structured Clinical Interview for DSM-IV Axis I disorders (SCID-I) [19]. Diagnostic reliability was found satisfactory with overall agreement for DSM-IV diagnostic categories of overall Kappa score 0.77 (95\% CI: 0.60-0.949). Duration of untreated psychosis (DUP) was established at baseline and measured in weeks from onset of psychosis until start of adequate treatment, with psychosis defined as the Positive and Negative Syndrome Scale (PANSS) [20] positive items P1, P3, P5, P6 and G9 equal to or above 4 for more than a week [21]. Symptoms at baseline and 1-year follow-up were measured with the PANSS using Wallwork's PANSS - five factor model consisting of a subset of items constituting positive, negative, disorganised, excited and depressive symptoms [22]. This model has been found to be ideal for assessment of first episode psychosis populations [23]. Substance abuse or dependence the last 6 months was established at baseline and 1-year follow-up using the scale Evaluating Substance Abuse in Persons with Severe Mental Disorders [24]. To measure substance use in general at baseline and 1-year follow-up, not only at the level of abuse and dependence, Alcohol Use Disorder Identification Test (AUDIT) [25] and Drug Use Disorder Identification Test (DUDIT) [26], was used. Satisfaction with life at baseline and 1-year follow-up was measured with the generic "Satisfaction with life in general" item in the Lehman's quality of life Interview, brief version [27, 28]. This single generic item was used since it has been found to be highly correlated with the 5 factors that are part of the subjective quality of life sub-scale [27]. Life satisfaction in general is rated from 1 to 7 , with 1 representing very satisfied with life, and 7 representing very unsatisfied with life.

\subsection{Functioning assessment}

Premorbid functioning was measured at baseline with the Premorbid Adjustment Scale (PAS) [29]. The scale was divided into a social- and an academic domain. For each of the domains we discriminated between the childhood scores and the difference between childhood score and the latest available score (change) [30].

Global functioning was measured at baseline and 1-year follow-up with the clinician-rated Global Assessment of Functioning scale Split version - function subscale (GAF-F), which focuses on overall degree of social and occupational functioning [31]. The sub-scale is rated from 1 to 100 with " 100 " representing the hypothetically best possible functioning and " 1 " representing the hypothetically lowest possible functioning.

Self-rated disability was measured at baseline and 1-year follow-up with the WHODAS 2.0, which is culturally sensitive, and reported to be reliable and valid to use across different populations of mental and physical disorders [9], including psychotic disorders [10]. Thirty-six statements are rated in terms of how much difficulty the participants have experienced with these statements during the last 4 weeks, on a 5 point Likert-type scale $(1=$ none, $2=$ mild, $3=$ moderate, $4=$ severe, $5=$ extreme). The statements are divided into six activity domains including: 1. Cognition: understanding and communicating. 2. Mobility: moving and getting around. 3. Self-care: attending to one's hygiene, dressing, eating and staying alone. 4. Getting along: interacting with people. 5. Life activities: household and work/school, and 6. Participation: joining in community activities, participating in society. The 4 work/school related items are only rated if the participant is working, consequently less than half of our sample responded to these items. As advised by the WHODAS 2.0 manual, the work/school items were therefore excluded from all analyses. The present study used the WHO complex scoring based on item-response-theory (IRT), which includes an algorithm that weights items according to their multiple levels of difficulty (Ustun et al. 2010 -WHODAS 2.0 manual). This provides 6 domain scores and a single summary score converted into a metric ranging from 0 to 100, where "0" represents no disability. The WHO also provides cross-cultural general population norms, based on large and representative population samples from 10 countries around the world (Ustun et al., 2003). This allows the disability summary score in the FEP sample to be matched with percentiles for the general population sample.

Clinically significant disability was defined as a summary score 1 standard deviation above the average disability of the WHO general population sample, thus at the level of the $16 \%$ most disabled. This equalled a summary score above 22 .

\subsection{Statistical analysis}

The statistical package for Social Sciences (IBM SPSS Statistics 22) was used. Parametric analyses were used for normally distributed variables, while non-parametric analyses were used for variables with skewed distributions. Analyses were two-tailed with the significance level set to 0.05 .

Degree of self-rated disability (WHODAS 2.0) in participants with first treated episode of psychosis at baseline and 1-year follow-up was computed by using the WHO complex scoring (IRT) algorithm providing 6 domain scores and a summary score. Rate of participants with clinically significant disability was calculated at baseline and 1-year follow-up. Changes in self-rated disability from baseline to 1-year follow-up, was investigated by Wilcoxon Signed Ranks Test. The relationship between self-rated disability and clinician-rated global functioning, self-rated life satisfaction and symptomatology at 1-year follow-up was investigated with Spearman correlations. The strengths of the correlations were based on Cohen [32]. Finally, baseline predictors of self-rated disability at 1-year follow-up was investigated with hierarchical multiple regression analysis (blockwise). Preliminary analysis (MannWhitney U and Spearman correlations) was carried out to identify premorbid and baseline demographic and clinical variables that were significantly related with the disability summary score at 1-year follow-up and therefore included in the regression analysis. None of the baseline categorical variables were included in the regression analysis because disability was not significantly different between the groups in any of these variables (not shown). The significantly associated baseline continuous variables were entered into the equation in five blocks based on a theoretical hypothesis of lifetime appearance: 1 ) social childhood $\left(0.279^{* *}\right)$ and academic childhood $\left(0.259^{* *}\right) ; 2$ ) years of education $\left(-0.270^{* *}\right)$; 3) DUP $\left(0.333^{* *}\right)$; 4) GAF-F $\left(-0.294^{* *}\right)$ and life satisfaction ( $\left.0.331^{* *}\right)$; and 5$)$ PANSS positive symptoms $\left(0.247^{* *}\right)$, PANSS excited symptoms $\left(0.241^{* *}\right)$ and PANSS depressive symptoms $\left(0.408^{* *}\right)$. The final models were examined for violations of assumptions underlying linear regression. Because of a skewed distribution, DUP was log transformed $($ DUPln +1$)$.

\section{Results}

Table 1 presents premorbid, demographic and clinical data for baseline and 1-year follow-up. The diagnostic distribution at 1-year follow-up was schizophrenia $=64.3 \%$, schizoaffective disorder $=$ $4.3 \%$, schizofreniform $=9.6 \%$ and other psychosis $=21.7 \%$.

\subsection{Self-rated disability in first treated episode of psychosis across the first year of treatment}

Self-rated disability for the participants with first treated episode of psychosis was high with an average disability summary score of 36.9 at baseline and 26.1 at 1-year follow-up on a scale where " 0 " represents no disability (Table 2.). According to WHO norms the average disability 
Table 1

Premorbid, demographic and clinical characteristics at baseline and 1-year follow-up.

\begin{tabular}{|c|c|c|}
\hline & $\begin{array}{l}\text { Baseline } \\
(n=115)\end{array}$ & $\begin{array}{l}1 \text { year follow-up } \\
(\mathrm{n}=115)\end{array}$ \\
\hline & Mean (SD) & Mean (SD) \\
\hline \multicolumn{3}{|l|}{ Demographics } \\
\hline Gender $n$ (\% males) & $75(65.2)$ & \\
\hline Age (years) & $26.2(6.8)$ & \\
\hline Education (years) & $12.1(2.0)^{\mathrm{e}}$ & \\
\hline IQ (WASI) & $102.7(14.2)$ & \\
\hline Minority background $n(\%)$ & $35(30.4)$ & \\
\hline \multicolumn{3}{|l|}{ Premorbid function ${ }^{\mathrm{a}}$ median (range) } \\
\hline PAS-social functioning childhood & $2.0(0-6)$ & \\
\hline PAS-social functioning change & $0.75(-3.5-5)$ & \\
\hline PAS-academic functioning childhood & $2(0-6)$ & \\
\hline PAS-academic functioning change & $0.5(-2.5-4)$ & \\
\hline DUP $^{\mathrm{b}}$ median (range) & $50(1-1300)$ & \\
\hline \multicolumn{3}{|l|}{ Clinical symptoms ${ }^{\mathrm{C}}$ mean $(S D)$} \\
\hline PANSS-positive symptoms & $10.7(3.9)$ & $8.7(4.2)$ \\
\hline PANSS-negative symptoms & $12.8(5.7)$ & $12.0(5.5)$ \\
\hline PANSS-disorganised symptoms & $5.6(2.3)$ & $5.6(2.3)$ \\
\hline PANSS-excited symptoms & $6.1(2.1)$ & $3.8(1.2)$ \\
\hline PANSS-depressive symptoms & $8.8(3.1)$ & $7.4(3.1)$ \\
\hline \multicolumn{3}{|l|}{ Medication $n(\%)$} \\
\hline Antipsychotic & $91(79)$ & $85(74)$ \\
\hline Antidepressant & $33(29)$ & $33(29)$ \\
\hline Antiepileptic & $6(5)$ & $6(5)$ \\
\hline \multicolumn{3}{|l|}{ Substance abuse } \\
\hline Drake-alcohol abuse/dep. $n(\%)$ & $14(12.2)$ & $10(8.7)$ \\
\hline Drake-drug abuse/dep. $n(\%)$ & $25(22.1)$ & $16(13.9)$ \\
\hline AUDIT-alcohol use median (range) & $7.6(7.3)$ & $7.5(6.9)$ \\
\hline DUDIT-drug use median (range) & $6.5(10.0)$ & $4.3(7.9)$ \\
\hline \multicolumn{3}{|l|}{ Global functioning } \\
\hline GAF-F & $43.9(13.2)$ & $52.1(15.3)$ \\
\hline \multicolumn{3}{|l|}{ Satisfaction with life } \\
\hline Life satisfaction in general & $4.40(1.52)^{d}$ & $3.60(1.39)$ \\
\hline \multicolumn{3}{|c|}{$\begin{array}{l}\text { a PAS script; social functioning } n=114 \text {, academic functioning } \\
\text { b } \text { Duration of untreated psychosis log transformed - in weeks. } \\
\text { c } \text { PANSS five factor model by Wallwork (2012). } \\
\text { d } n=110 . \\
\text { e } n=113 .\end{array}$} \\
\hline
\end{tabular}

summary score (median) at baseline was at the 91 st percentile, while at 1 -year follow-up it was at the 86th percentile. This indicates an average self-rated disability across the first year after treatment initiation in first treated episode of psychosis around the $10 \%$ highest level of disability in the WHO general population sample. At 1 -year follow-up, 7 participants $(6,1 \%)$ in the present sample had a disability summary score under the 50th percentile of the WHO sample, 2 participants (1.7\%) had a disability summary score between the 50th and 59th percentiles, 9 participants (7.8\%) had a disability summary score between 60th and 69th percentiles, 15 participants (13\%) had a disability summary score between the 70th and 79th percentiles, 49 participants (40\%) had a disability summary score between 80th and 89th percentiles, and 36 participants (31.3\%) had a disability summary score above the 90th percentile.

With clinically significant disability being defined as a summary score 1 standard deviation above the average of the WHO sample,

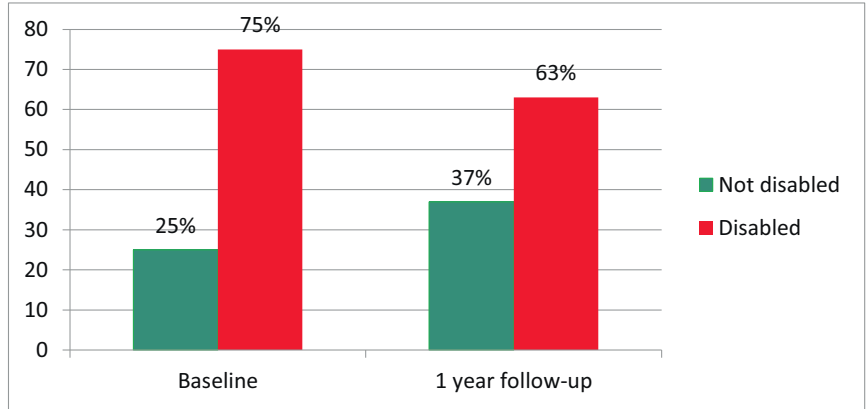

Fig. 2. Rate of clinically significant self-rated disability ${ }^{\mathrm{a}}$ a. Clinically significant disability $=$ whodas 2.0 summary score $1 \mathrm{SD}$ above the who general population average (i.e. summary score $>22$ ).

thus at the level of the $16 \%$ most disabled, $75 \%$ of the present sample were disabled at baseline and $63 \%$ at 1 -year follow-up. In other words, as presented in Fig. 2, 37\% of the sample rated themselves not to be disabled at a level defined as clinically significant at 1-year follow-up.

The disability domain scores are presented in Table 2 and Fig. 3. At baseline and 1-year follow-up the highest level of disability was in two social domains: "Getting along with people" and "Participation in society", followed by "Understanding and communication" and "Life activities (Household)". A lower level of disability was found in the domains "Getting around" and "Self-care".

As presented in Table 2 and Fig. 3, all the disability domains apart from "Life activities", improve significantly (thus decrease) from baseline to 1-year follow-up.

3.2. Relationship between self-rated disability and clinician-rated global functioning, self-rated life satisfaction and symptomatology in first treated episode of psychosis at 1 year follow-up

The relationship between self-rated disability and clinician-rated global functioning, self-rated life satisfaction and symptomatology at 1-year follow-up is presented in Table 3. Firstly, the self-rated disability summary score and all the disability domains had significant correlations, with weak to medium strength, with the clinician-rated global functioning score (GAF-F). The two social domains "Getting along with people" and "Participation in society" had the strongest association. Secondly, the disability summary score and all the disability domains had significant correlations, of a mainly medium and strong strength, with self-rated life satisfaction. Again the two social domains "Getting along with people" and "Participation in society" had the highest associations, along with "Understanding and communicating". Thirdly, the self-rated disability summary score had a significant correlation with a strong strength with depressive symptoms, medium strength with positive symptoms, and a weak strength with disorganised symptoms, yet no significant associations with negative and excited symptoms. All disability domains significantly correlated, with a mainly strong and medium strength, with depressive symptoms.

Table 2

Average self-rated disability domain scores and summary score ${ }^{\mathrm{a}}$ at baseline and 1-year follow-up.

\begin{tabular}{|c|c|c|c|c|}
\hline & $\begin{array}{l}\text { Baseline } \\
(\mathrm{n}=115)\end{array}$ & $\begin{array}{l}1 \text { year follow-up } \\
(\mathrm{n}=115)\end{array}$ & Wilcoxon signed ranks test & \\
\hline Disability domain scores ${ }^{\mathrm{a}}$ & Median (range) & Median (range) & $\mathrm{Z}$ & $P$ \\
\hline Understanding and communicating & $35.0(1-110)$ & $25.0(0-100)$ & $\mathrm{z}=-3.70$ & $\mathrm{P}<.0005$ \\
\hline Getting around & $12.5(0-125)$ & $0.25(0-93.3)$ & $z=-3.09$ & $P=.002$ \\
\hline Self-care & $10.0(0-120)$ & $0(0-100)$ & $\mathrm{z}=-3.81$ & $P<.0005$ \\
\hline Getting along with people & $58.33(0-158)$ & $41.6(0-158.3)$ & $Z=-3.35$ & $P=.001$ \\
\hline Life activities-household & $30.0(0-160)^{\mathrm{b}}$ & $25(0-160)$ & $\mathrm{z}=-1.69$ & $P=.091$ \\
\hline Participation in society & $47.9(4.1-129.1)$ & $33.3(0-120)$ & $z=-4.62$ & $\mathrm{P}<.0005$ \\
\hline Disability summary score ${ }^{a}$ & $36.9(5.4-126.0)$ & $26.1(0-104.3)$ & $\mathrm{z}=-5.05$ & $\mathrm{P}<.0005$ \\
\hline
\end{tabular}

a WHODAS 2.0 complex scoring (IRT). 


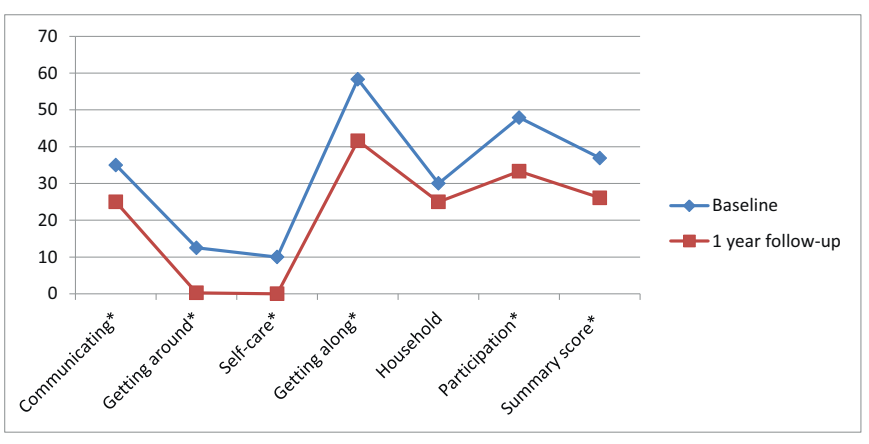

Fig. 3. Change in average self-rated disability domain scores and summary scores from baseline to 1-year follow-up (Wilcoxon signed ranks test) a. whodas 2.0 complex scoring (IRT) * Significant change from baseline to 1-year follow-up.

All disability domains significantly correlated with a weak or medium strength with positive symptoms, while only "self-care" and "Participation in society" had significant correlations with a weak strength with disorganised symptoms. "Understanding and communicating" was the only domain that significantly correlated with a weak strength with negative symptoms, while, none of the disability domains correlated with excited symptoms. Thus, depressive symptoms had the highest correlations with self-rated disability. And again the two social domains "Getting along with people" and "Participation in society", as well as "Understanding and communicating", had the highest associations with symptoms.

\subsection{Premorbid and baseline predictors of self-rated disability in first treated episode of psychosis at 1-year follow-up}

The bivariate analyses between self-rated disability summary score at 1-year follow-up and the significantly associated premorbid and baseline variables were as follows in the five blocks in the regression analysis: 1) social adjustment childhood $\left(0.269^{* *}\right)$ and academic adjustment childhood $\left(0.275^{* *}\right)$; 2) years of education $\left(-0.273^{*}\right)$; 3) DUP $\left(0.286^{*}\right)$; 4) GAF-F $\left(-0.284^{* *}\right)$ and life satisfaction $\left(0.310^{* *}\right)$; and 5) PANSS positive symptoms $\left(0.177^{*}\right)$, PANSS excited symptoms $\left(0.220^{* *}\right)$ and PANSS depressive symptoms $\left(0.389^{* *}\right)$. In the subsequent hierarchical multivariate regression analyses, social and academic childhood adjustment, DUP, baseline GAF-F, baseline life satisfaction and baseline depressive symptoms significantly predicted disability at 1-year follow-up when they were entered into the equation in their corresponding blocks. However, as presented in Table 4, in the final block only increase in baseline depressive symptoms (beta $=0.286$ ) was a significant predictor of higher disability, with increase in DUP reaching a subthreshold level of significance (beta $=0.163$ ) (Table 4.). The final model as a whole was significant $(\mathrm{F}(9,99)=5.121, p<.0005)$, explaining a total of $25.6 \%$ of the variance in disability.

\section{Discussion}

The main findings include that self-rated disability in first treated episode of psychosis was high, especially in social domains. However, the level of disability improved across the first year of treatment, with $37 \%$ not rating themselves disabled at a clinically significant level at 1-year follow-up. Secondly, self-rated disability was more closely related to life satisfaction in general and depressive symptoms than to clinician-rated global functioning and positive symptoms at 1-year follow-up. Finally, self-rated disability at 1 year follow-up was predicted by baseline level of depression. To our knowledge this is the first report of self-rated disability and how it relates to other outcome measures, across the first year of treatment in first episode psychosis.

Our first set of findings show a high average level of disability compared to the WHO cross-cultural general population sample. At baseline and 1-year follow-up the average disability summary scores in the present sample were around the 90th percentile for the WHO general population norm, thus at the same level as the $10 \%$ with highest disability in the general population. Rate of disability considered to be clinically significant in our first treated episode of psychosis sample was $76 \%$ at baseline and $63 \%$ at 1 -year follow-up. In other words, $37 \%$ of the sample did not consider themselves to be disabled at a clinically significant level at 1-year follow-up. Comparatively, in a previous study based on clinician-ratings, $26 \%$ of the first episode psychosis sample was in symptomatic remission [33] at 1-year follow-up, while $14 \%$ were in early clinical recovery defined as symptomatic remission; GAF-F $\geq 60 ; 50 \%$ work/study; and independent living [15]. Taken together, this suggests that rate of participants not considering themselves as disabled is higher than participants considered in clinical recovery as rated by clinicians. This is in line with reports of a higher rate of subjective experience of recovery, also called personal recovery, compared to objective level of clinical recovery [34].

The fact that the highest level of self-rated disability was reported in the social domains "Getting along with people" and "Participation in society", is in line with previous studies, including the study of FEP [10-13]. However, the social domain scores in the present first treated episode of psychosis study show a higher level of disability than that found in the two groups of older patients and outpatients with schizophrenia in two previous studies $[10,11]$. This may be due to the fact that older and more chronic patients with schizophrenia have adapted to their health challenges and situation, compared to the relatively young participants in the present study, whom are perhaps still comparing themselves to their peers. Moreover, the older patients in the previous study lived in board and care settings, where they are likely to have far more help and support than the younger participants in the present study whom mainly live outside community care. The present findings show a low level of self-rated disability in the "Getting around" and "Self-care" domains, which is what we would expect from a sample with mental illness as opposed to a sample with physical illness. These findings also show that self-rated disability improved from baseline to 1-year follow-up on all domains apart from "Life activities". This is in

Table 3

Relationship between self-rated disability ${ }^{\mathrm{a}}$ and clinician-rated function, self-rated life satisfaction and symptoms at 1-year follow-up (Spearman correlation).

\begin{tabular}{|c|c|c|c|c|c|c|c|}
\hline & GAF-F & Life satisfaction $^{\mathrm{d}}$ & PANSS-positive ${ }^{\mathrm{b}}$ & PANSS-negative ${ }^{\mathrm{b}}$ & PANSS-disorganised ${ }^{\mathrm{b}}$ & PANSS-excited $^{b}$ & PANSS-depressive ${ }^{\mathrm{b}}$ \\
\hline \multicolumn{8}{|l|}{ Disability domain scores ${ }^{\mathrm{a}}$} \\
\hline Understanding and communicating & $-0.373^{* *}$ & $0.581^{* *}$ & $0.351^{* *}$ & $0.188^{*}$ & 0.180 & 0.054 & $0.520^{* *}$ \\
\hline Getting around & $-0.402^{* *}$ & $0.369^{* * *}$ & $0.259^{* *}$ & 0.131 & 0.181 & 0.012 & $\mathbf{0 . 3 3 1} 1^{* *}$ \\
\hline Self-care & $-0.281^{*}$ & $0.254^{* *}$ & $0.220^{* *}$ & 0.042 & $0.202^{*}$ & 0.133 & $0.257^{* *}$ \\
\hline Getting along with people & $-0.473^{* *}$ & $0.532^{* *}$ & $0.366^{* *}$ & 0.124 & 0.181 & 0.104 & $0.466^{* *}$ \\
\hline Life activities - household & $-0.231^{*}$ & $\mathbf{0 . 3 1 8}^{* *}$ & $0.218^{* *}$ & 0.044 & 0.041 & 0.145 & $0.337^{* *}$ \\
\hline Participation in society & $-0.427^{* *}$ & $0.484^{* *}$ & $0.354^{* *}$ & 0.025 & $0.272^{* *}$ & 0.115 & $0.537^{* *}$ \\
\hline Disability summary score ${ }^{a}$ & $-0.477^{* *}$ & $0.551^{* *}$ & $0.387^{* *}$ & 0.106 & $0.241^{* *}$ & 0.145 & $0.535^{* *}$ \\
\hline
\end{tabular}

\footnotetext{
a WHODAS 2.0
}

b PANSS five factor model by Wallwork (2012),

c Global Assessment of Functoning Scale - Functioning (Split version).

d Generic item from Lehman's quality of life interview, brief version. 
Table 4

Premorbid and baseline demographic and baseline clinical predictors of self-rated disability summary score ${ }^{\mathrm{a}}$ at 1-year follow-up (Hierarchical multiple regression analysis).

\begin{tabular}{|c|c|c|c|c|c|c|c|c|c|}
\hline & \multicolumn{5}{|c|}{ Contribution of each variable in block 5} & \multicolumn{4}{|c|}{ Model summary for each block } \\
\hline & $B$ & S.E. ${ }^{d}$ & Beta & $t$ & $p$ & & R square & Adjusted R square & $p$ \\
\hline Social adjustment childhood & 1.996 & 1.247 & 0.145 & 1.601 & 0.113 & Block 1 & 0.114 & 0.097 & 0.002 \\
\hline Academic adjustment childhood & 0.551 & 1.683 & 0.034 & 0.327 & 0.744 & & & & \\
\hline Education (years) & -1.723 & 1.063 & -0.155 & -1.621 & 0.108 & Block 2 & 0.144 & 0.120 & 0.055 \\
\hline DUPIn ${ }^{\mathrm{b}}$ & 2.194 & 1.203 & 0.163 & 1.825 & 0.071 & Block 3 & 0.190 & 0.159 & 0.017 \\
\hline GAF-FC & -0.189 & 0.173 & -0.109 & -1.093 & 0.277 & Block 4 & 0.246 & 0.202 & 0.026 \\
\hline Life satisfaction $^{\mathrm{c}}$ & 1.089 & 1.541 & 0.072 & 0.707 & 0.481 & & & & \\
\hline PANSS positive symptoms ${ }^{c}$ & 0.099 & 0.529 & 0.017 & 0.188 & 0.852 & Block 5 & 0.318 & 0.256 & 0.019 \\
\hline PANSS excited symptoms ${ }^{c}$ & 0.601 & 0.964 & 0.057 & 0.623 & 0.534 & & & & \\
\hline PANSS depressive symptoms ${ }^{c}$ & 2.091 & 0.702 & 0.286 & 2.979 & 0.004 & & & & \\
\hline
\end{tabular}

a WHODAS 2.0

b DUPln = Duration of untreated psychosis log transformed.

c Baseline.

d S.E. $=$ Standard error.

line with previous outcome studies, reporting improvement in clinician-rated global functioning the first year of treatment [2], and improvement in life satisfaction in general the two first years of after treatment [16].

Our second set of findings on the relationship between self-rated disability and other outcome measures at 1-year follow-up includes weak and moderate significant correlations between self-rated disability measured by WHODAS 2.0 and clinician-rated functioning measured by GAF-F. This is in line with previous studies [12], and suggests that the two scales measure related but different phenomena. If these findings are a reflection of clinicians believing that their patients are more disabled than the patients themselves do, this could impact negatively on the patients' recovery process by dampening their sense of mastery, empowerment and hope for the future.

At 1-year follow-up self-rated life satisfaction measured with the satisfaction with life in general item in Lehman's Quality of Life interview, had mainly medium and strong significant correlations with various aspects of self-rated disability. This is also in line with previous studies $[10,12]$, and suggests that the two scales measure related, but distinct and complementary phenomena. This may also suggest that self-rated disability, and especially social disability, is more closely related with life satisfaction than global functioning. Alternatively, it may reflect that self-report measures are more closely related. Nevertheless, these findings imply that it is important to measure self-rated disability in addition to clinician-rated global functioning and self-rated life satisfaction in order to capture the subjective experience of disability in people with psychotic disorders.

That self-rated disability has stronger significant associations with depressive symptoms than positive symptoms at 1-year follow-up, is in line with the previous study that includes an older sample with schizophrenia [10], and the previous first episode psychosis study [13]. Moreover, the associations were highest for the social domains "Getting along with people" and "Participation in society", as well as "Understanding and communicating". This indicates a close relationship between the experience of social disability and depression, although, the direction of this relationship is uncertain. It could imply that an increase in depression leads to increase in social disability, or to a more pessimistic scoring of social disability. Alternatively, the finding may reflect that a high social disability leads to an increase in depressive symptoms. Nevertheless, the significance of depression reflects the high level of depression in first episode psychosis, being present in 50\% at treatment initiation [35] and 35\% at one year follow-up [36]. The only disability domain that was associated with negative symptoms was "Understanding and communicating" which largely represents cognition, which is in line with studies finding a close relationship between negative symptoms and cognitive impairment [37]. However, it may be that disability would be more closely related to a measure of negative symptoms that is more detailed and precise than the PANSS.
Our third set of findings include that self-rated disability at 1-year follow-up was only significantly predicted by level of depressive symptoms at baseline, which is in line with the previous study of older patients [10]. This adds to the above discussion regarding the close relationship between depression and self-rated social disability, suggesting that depression might be the driving force. In contrast, clinician-rated symptomatic remission at 1 -year follow-up was significantly predicted by baseline positive symptoms in a previous study [15]. Therefore, we can speculate that while baseline psychotic symptoms are important for clinical recovery, depression is more important for personal recovery, although this obviously requires further research. Finally, self-rated disability at 1 -year follow-up was predicted by DUP at a subthreshold level, consistent with earlier findings reporting that DUP is important for outcome in first episode psychosis [15,38].

There are several clinical implications of these findings. The participants in this study were recruited from various in-patient and out-patient units across Oslo, and received treatment on an individual basis, based on the Norwegian treatment guidelines for first episode psychosis. However, because of the close relationship between depression and social disability, the findings imply that specialised treatment should target depression alongside psychotic symptoms the first year of treatment in first episode psychosis. Moreover, specialised treatment of social disability, such as social skills training, social cognition training and cognitive behaviour therapy (CBT), all aimed at improving social ability and thus integration, is also important the first year of treatment in first episode psychosis. Recovery-focused CBT interventions targeting disability broadly, including activity and participation domains are recommended [39]. In line with the ICF, contextual factors that interact with symptoms and result in social disability should also be a target in treatment. Thus psychosocial interventions directed at the environment or context surrounding people with FEP are also needed in order to increase social integration. Moreover, the findings imply that there are signs of recovery already the first year after treatment start, adding to the literature that provides hope for recovery in this patient group $[5,34]$. Finally, the scientific implications of this study are that it is important to assess self-rated disability as well as clinician-rated global functioning or self-rated life satisfaction, in order to fully assess outcome in FEP.

A limitation to the present study could be that the reliability of the self-report may have been compromised by cognitive impairment and limited insight common amongst people with psychotic disorders. However, the purpose was to capture the qualitative subjective experience of disability irrespective of level of cognitive function or insight. The fact that the sample is recruited through the public mental health care system in a country with a catchment-area patient admittance system should result in the sample being highly representative of the treated patient population. However, there are two issues that limit the extent to which we can draw this conclusion. Firstly, due to 
regulations by the Norwegian Ethical board we had no data on the number of people that did not consent to participate in the study. Secondly, $38 \%$ of the participants that entered into the study at baseline were not reassessed at 1 -year follow-up, and 53 participants were excluded from the final sample due to missing data or exclusion criteria. Nevertheless, the sample size in the present study is relatively large compared to previous studies using WHODAS 2.0 comprising considerably smaller samples of around 20 participants [13]. Future research should investigate environmental factors that influence self-rated disability in first episode psychosis over time in order to guide the further development of early treatment and environmental facilitation.

\subsection{Conclusion}

In sum, the present study suggests that self-rated disability in first treated episode of psychosis is high, but improves across the first year, indicating signs of early recovery. Moreover, self-rated disability is related, but distinct from clinician-rated global functioning and self-rated life satisfaction, suggesting that assessment of self-rated disability is also required in order to fully describe outcomes in first episode psychosis. Finally, the findings highlight the need for specialised treatment of depression and social disability in the early phases of first episode psychosis.

\section{Acknowledgements}

The authors are very grateful to the study participants for their time and effort devoted to participation.

\section{Funding}

This work was supported by grants from the South-Eastern Norway Regional Health Authority (\#2006258, \#2015-088), the Research Council of Norway (\#217776) through its Centre of Excellence funding scheme (\#223273), and the KG Jebsen Foundation (\#SKGJ_MED_008). The funding sources had no involvement in the study design, data collection, analysis and interpretation of data, in the writing of the manuscript or in the decision to submit the paper for publication.

\section{Declaration of conflict}

OAA received speaker's honorarium from Lundbeck. All other authors reported nothing to declare.

\section{References}

[1] Stouten LH, et al. Psychosocial functioning in first-episode psychosis and associations with neurocognition, social cognition, psychotic and affective symptoms. Early Interv Psychiatry 2015;11(1):23-36.

[2] Faerden A, et al. Apathy, poor verbal memory and male gender predict lower psychosocial functioning one year after the first treatment of psychosis. Psychiatry Res 2013;210(1):55-61.

[3] Niendam TA, Jalbrzikowski M, Bearden CE. Exploring predictors of outcome in the psychosis prodrome: implications for early identification and intervention. Neuropsychol Rev 2009;19(3):280-93.

[4] Jaaskelainen E, et al. A systematic review and meta-analysis of recovery in schizophrenia. Schizophr Bull 2013:39(6):1296-306.

[5] Leamy $M$, et al. Conceptual framework for personal recovery in mental health: systematic review and narrative synthesis. Br J Psychiatry 2011;199(6):445-52.

[6] Gardsjord ES, et al. Subjective quality of life in first-episode psychosis. A ten year follow-up study. Schizophr Res 2016;172(1-3):23-8.
[7] Switaj P, et al. Disability and schizophrenia: a systematic review of experienced psychosocial difficulties. BMC Psychiatry 2012;12:193.

[8] World Health Organization. International classification of functioning, disability and health: ICF. Geneva: World Health Organization; 2001.

[9] Ustun TB, et al. Developing the World Health Organization disability assessment schedule 2.0. Bull World Health Organ 2010;88(11):815-23.

[10] McKibbin C, Patterson TL, Jeste DV. Assessing disability in older patients with schizophrenia: results from the WHODAS-II. J Nerv Ment Dis 2004;192(6):405-13.

[11] Ertugrul A, Ulug B. The influence of neurocognitive deficits and symptoms on disability in schizophrenia. Acta Psychiatr Scand 2002;105(3):196-201.

12] Chopra PK, Couper JW, Herrman H. The assessment of patients with long-term psychotic disorders: application of the WHO disability assessment schedule II. Aust N Z J Psychiatry 2004;38(9):753-9.

[13] Chudleigh C, et al. How does social functioning in the early stages of psychosis relate to depression and social anxiety? Early Interv Psychiatry 2011;5(3):224-32.

[14] Velthorst E, et al. Disability in people clinically at high risk of psychosis. Br J Psychiatry 2010;197(4):278-84.

[15] Simonsen C, et al. Early clinical recovery in first-episode psychosis: symptomatic remission and its correlates at 1-year follow-up. Psychiatry Res 2017;254:118-25.

[16] Melle I, et al. The development of subjective quality of life over the first 2 years in first-episode psychosis. J Nerv Ment Dis 2010;198(12):864-9.

[17] Eack SM, Newhill CE. Psychiatric symptoms and quality of life in schizophrenia: a meta-analysis. Schizophr Bull 2007;33(5):1225-37.

[18] Secher RG, et al. Ten-year follow-up of the OPUS specialized early intervention tria for patients with a first episode of psychosis. Schizophr Bull 2015;41(3):617-26.

[19] First, M., Spitzer, R., Gibbon, M., Williams, J. B. W., Structured clinical interview for DSM-IV axis I disorders: patient edition (SCID-P), version 2. 1995, New York: New York State Psychiatric Institute, Biometrics Research.

[20] Kay SR, Fiszbein A, Opler LA. The positive and negative syndrome scale (PANSS) for schizophrenia. Schizophr Bull 1987;13(2):261-76.

[21] Larsen TK, Johannessen JO, Opjordsmoen S. First-episode schizophrenia with long duration of untreated psychosis. Pathways to care. Br J Psychiatry Suppl 1998;172(33): 45-52.

[22] Wallwork RS, et al. Searching for a consensus five-factor model of the positive and negative syndrome scale for schizophrenia. Schizophr Res 2012;137(1-3): 246-50.

[23] Langeveld J, et al. Is there an optimal factor structure of the positive and negative syndrome scale in patients with first-episode psychosis? Scand J Psychol 2013; 54(2):160-5.

[24] Drake RE, Mueser KT, McHugo GJ. Clinician rating scales. In: Sederer L, Dickey IB, editors. Outcomes assessment in clinical practice. Baltimore, MD: Williams \& Wilkins; 1996. p. 113-6.

[25] Saunders JB, et al. Development of the alcohol use disorders identification test (AUDIT): WHO collaborative project on early detection of persons with harmful alcohol consumption-II. Addiction 1993;88(6):791-804.

[26] Berman AH, et al. Evaluation of the drug use disorders identification test (DUDIT) in criminal justice and detoxification settings and in a Swedish population sample. Eur Addict Res 2005;11(1):22-31.

[27] Melle I, et al. Measuring quality of life in first-episode psychosis. Eur Psychiatry 2005;20(7):474-83.

[28] Lehman AF. A quality of life interview for the chronically ill. Eval Program Plann 1988;11:51-62.

[29] Cannon-Spoor HE, Potkin SG, Wyatt RJ. Measurement of premorbid adjustment in chronic schizophrenia. Schizophr Bull 1982;8(3):470-84.

[30] Haahr U, et al. First-episode psychosis: diagnostic stability over one and two years. Psychopathology 2008;41(5):322-9.

[31] Pedersen G, Hagtvet KA, Karterud S. Generalizability studies of the global assessment of functioning-split version. Compr Psychiatry 2007;48(1):88-94.

[32] Cohen JW. Statistical power analysis for the behavioral sciences. 2nd ed. Hillsdale, NJ: Lawrence Erlbaum Associates; 1988.

[33] Andreasen NC, et al. Remission in schizophrenia: proposed criteria and rationale for consensus. Am J Psychiatry 2005;162(3):441-9.

[34] Warner R. Recovery from schizophrenia. Brunner-Routledge: London; 2004.

[35] Romm KL, et al. Depression and depressive symptoms in first episode psychosis. J Nerv Ment Dis 2010;198(1):67-71.

[36] Sonmez N, et al. Depressive symptoms in first episode psychosis: a one-year followup study. BMC Psychiatry 2013;13:106.

[37] Huang M, et al. Relationship between negative symptoms and neurocognitive functions in adolescent and adult patients with first-episode schizophrenia. BMC Psychiatry 2016;16(1):344.

[38] Ten Velden Hegelstad W, et al. Early detection, early symptom progression and symptomatic remission after ten years in a first episode of psychosis study. Schizophr Res 2013;143(2-3):337-43.

[39] Nowak I, et al. Disability and recovery in schizophrenia: a systematic review of cognitive behavioral therapy interventions. BMC Psychiatry 2016;16:228. 